\title{
An In-Vitro Study to Find the Antibiotic Susceptibility of Aggregatibacter Actinomycetocomitans to Various Antibiotics
}

\author{
B Manovijay*, R Saranyan, G Balaji Babu, A Anitha and I Nithya \\ Department of periodontics, Vinayaka mission's sankrachariyar dental college and hospital, India
}

Received: January 22, 2018; Published: February 02, 2018

*Corresponding author: B Manovijay, Department of periodontics, vinayaka missions sankrachariyar dental college and hospital, Ariyanoor, Salem, Tamilnadu, India, Tel: 9443600901; Email: drmanovijaymds@yahoo.com

\section{Abstract}

Aim: Aggregatibacter actinomycetocomitans,a gram negative facultative anaerobic coccobacilli is the main etiologic agent in the pathogenesis of localized aggressive periodontitis. So considering this, the aim of the present study was to study the sensititivity of the organism to various drugs in vitro.

Materials and Methods: Microbial plaque samples from 40 patients who were diagnosed to have localized aggressive periodontitis were cultured anaerobically to confirm Aggregatibacter actinomycetocomitans. Doycycline, amoxicillin, kanamycin, norfloxacin, ciprofloxacin, gentamycin, netilmycin co-trimoxazole, metronidazole, and ampicillin and cephazolin antibiotic sensitivity discs were placed in the culture plate. The plates were incubated anaerobically at 37 degree Celsius for 48 hours. At the end of the duration the plates were opened and the sensitivity of the organism to various drugs was examined.

Results: The antibiotic sensitivity test showed that the organism was found to be highly sensitive to tetracycline, amoxicillin, metronidazole, kanamycin, norfloxacin, ciprofloxacin, gentamycin and netilmycin. It was moderately sensitive to Co-Trimoxazole and resistant to ampicillin and Cephazolin.

Conclusion: The above results have shown the sensitivity of A.a to vaious drugs. Thus the synergisitic use of antibiotics along with periodontal surgery might improve the periodontal health in localised aggressive periodontitis patients.

Keywords: Aggregatibacter actinomycetocomitans; Aggressive periodontitis; Antibiotics; Sensitivity

\section{Introduction}

Aggressive periodontitis is a disease of the periodontium occurring in an otherwise healthy adolescent which is characterized by a rapid loss of alveolar bone about more than one tooth of the permanent dentition [1]. The amount of destruction manifested is not commensurate with the amount of local irritants. According to the international workshop of classification of periodontal diseases held in 1999, American Academy of Period ontology classified it in to localized and generalized forms. Aggregatibacter actinomycetocomitans (A.a), a gram negative facultative anaerobic rod is the main etiologic agent that is found in the diseased (incisors and molars) sites of localised aggressive periodontitis patients [2]. The other common features of localized aggressive periodontitis (LAP) include circum-pubertal onset, rapid bone loss on at least two permanent teeth (one being a permanent first molar), with two or fewer permanent teeth other than the first molars or incisors involved ,abnormalities in phagocytic function, hyper responsive monocytes and robust serum antibody titre to infecting agents [3]. Due to the increased resistance of the microorganisms to antibiotics, antibiotic susceptibility of periodontal pathogens should be carried out for better treatment and prognosis. Considering the above facts, the present study was designed to test the sensitivity of A.a to various antibiotics in vitro.

\section{Materials and Methods}

The present study was conducted in the department of periodontics, Rajah Muthiah Dental College and Hospital, Annamalai University, Chidambaram, Tamilnadu state, India. A total of 40 patients who were diagnosed to have localized aggressive periodontitis as per AAP classification were selected for the study according to the following inclusion criteria [2]. Patients chosen 
were free from any systemic diseases, had not undergone oral prophylaxis or any other periodontal treatment and had not taken antibiotics 6 months prior to the study. A microbial sampling was done for each subject. Plaque sampling was done at the first molar and the central incisor of the same side. The selected teeth were isolated with sterile cotton rolls. The loosely adherent plaque in direct proximity to the sample site was carefully removed using sterile cotton gauze. A sterile paper point was introduced in to the mesio-buccal inter proximal pocket until resistance was described by slots [4]. It was kept in place for 10 seconds and after removal the collected sample was then transferred into a test tube containing sterile saline. The bacterial deposits were dispersed in a vortex mixture for 60 seconds. Later the growth of A.a was confirmed from colony grown on Blood agar, Chocolate agar and Thioglycolate Blood agar plates. The following antibiotic sensitivity discs were placed on the culture plate. In one plate, commonly used antibiotics, such as doxycycline and amoxicillin were placed. In the other plates other antibiotic discs like kanamycin, norfloxacin, ciprofloxacin, gentamycin, netilmycin, co-trimoxazole, metronidazole, ampicillin and cephazolin were placed (Figure 1). The plates were incubated anaerobically at $37^{\circ} \mathrm{C}$ for 48 hours. At the end of the duration the plates were opened and sensitivity of the organism to various drugs was examined (Figure 2).

\section{Results}

(Figures 1-3) and (Table 1)

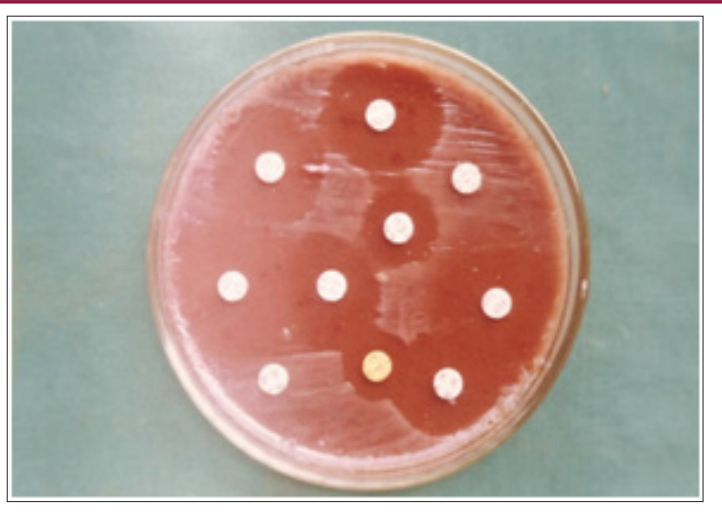

Figure 1: Showing the antibiotic various antibiotic discs placed in the culture media of Aggregatibacter actinomycetocomitans.

Table 1: Antibiotic susceptibility of Aggregatibacter actinomycetocomitans to various antibiotics.

\begin{tabular}{|c|c|c|}
\hline Highly sensitive & Moderately sensitive & Resistant \\
\cline { 1 - 1 } doxycycline, & & \\
\cline { 1 - 1 } amoxicillin, & & \\
\cline { 1 - 1 } metronidazole, & & \\
\cline { 1 - 1 } kanamycin, & Co-Trimoxazole & $\begin{array}{c}\text { ampicillin and } \\
\text { cephazolin }\end{array}$ \\
\cline { 1 - 1 } norfloxacin, & & \\
\cline { 1 - 1 } ciprofloxacin, & & \\
\cline { 1 - 1 } $\begin{array}{c}\text { gentamycin and } \\
\text { netilmycin }\end{array}$ & & \\
\hline
\end{tabular}

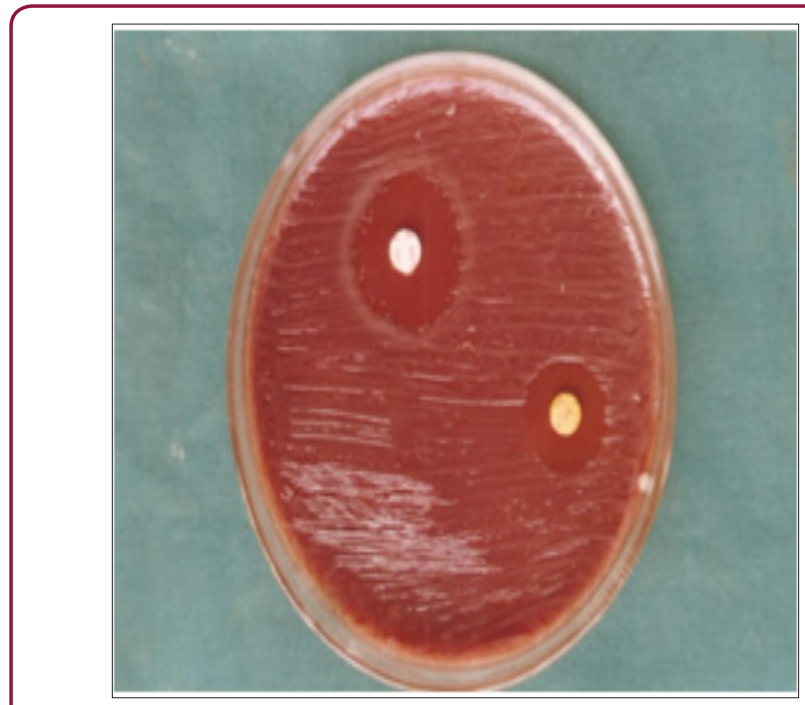

Figure 2: Showing the antibiotic sensitivity of A.a.

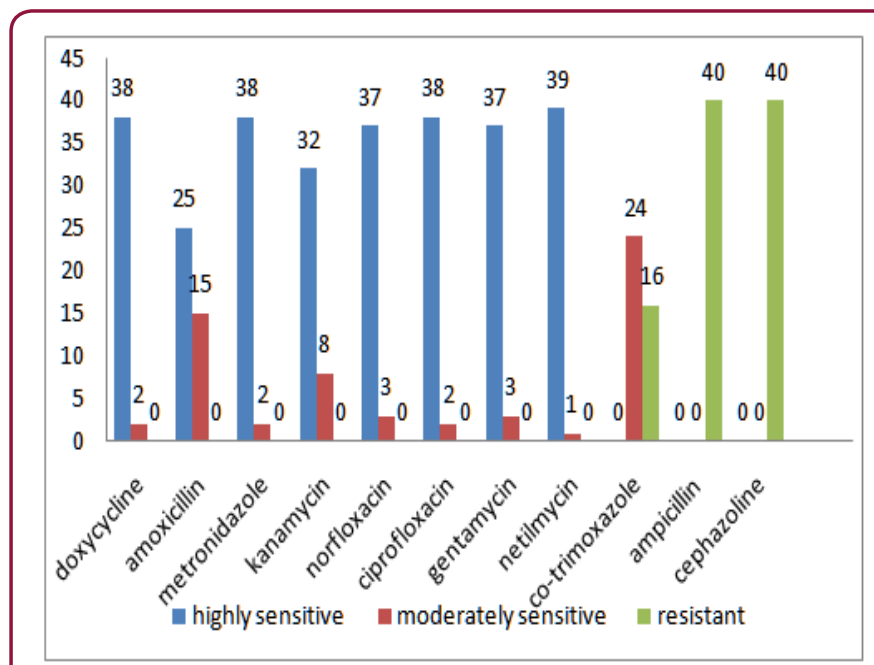

Figure 3: Sensitivity of A.a to various antibiotics.

\section{Discussion}

The major role in the treatment of periodontal disease is to arrest deterioration of the supporting periodontal disease and to allow for the establishment of new periodontal attachment. Since most forms of periodontal disease are apparently caused by the specific bacterial species, treatment should be directed towards the elimination of the periodontal pathogens. Various clinical data are available indicating that periodontal surgery along with systemic antimicrobial treatment could promote healing in LAP patients. Antimicrobial therapy, usually administered systemically, offers several advantages in the treatment of periodontal disease. Not only can it act against pathogens that have invaded soft tissue, but it can also inhibit microorganisms at other sites that are relatively inaccessible, for example, deep, narrow periodontal pockets. The literature describes a number of empirical protocols for the systemic antibiotic treatment of LAP.

These protocols are mostly based on doxycycline, a pharmacological derivative of tetracycline $[5,6]$ or the combination 
of amoxicillin and metronidazole [6,7]. In the present study also the organisms were highly sensitive to tetracycline and amoxicillin. Other protocols involve clindamycin [7], amoxicillin [8], or metronidazole $[9,10,11]$ as sole agents. There are currently no universally accepted protocols for the treatment of A. actinomycetocomitans-associated periodontitis. In a study done by Orit Oettinger-Barak [12] A. actinomycetocomitans JP2 homotypic biofilm were more susceptible in vitro to doxycycline than amoxicillin and metronidazole combination. Layton et al. have reported that amoxicillin penetrates well into the gingival crevicular fluid, and both achieve and maintain levels inhibitory for the majority of the four organisms cultivated from periodontal pockets [13]. Graevenitz et al and Holmes et al have shown in their study that Actinobacillus or Aggregatibacter strains were generally susceptible to a range of antibiotics, including cephalosporins, cefotaxime, cefazolin, doxycycline and aminoglycosides but in our study the organisms were resistant to cephazolin $[8,13]$.

It is recommended that empirical treatment with betalactams associated to fluorquinolones should be limited, since both groups of antibiotics activate common resistance mechanisms - thus favoring the appearavnce of resistances in important pathogens such as Pseudomonas aeruginosa and A.a species [14]. In a study by Veloo et al. geographical differences in the susceptibility profiles of Porphyromonas gingivalis and A. actinomycetocomitans between European countries were noted [15]. Aggregatibacter actinomycetocomitans appeared to be amoxicillin-resistant but failed to show beta-lactamase activity. In our study the organisms were highly sensitive to kanamycin and netilmycin but there were only limited studies in the literature supporting this fact. It may be due to the increased side effects of the drug than its beneficial effect. So further studies should be carried out to confirm the above fact. Comparison with a previous study from The Netherlands showed minor differences in susceptibility profiles, but the MIC90 values of A. actinomycetocomitans for amoxicillin, clindamycin, azithromycin and tetracycline were higher [15]. Takahashi et al. [16] showed reduced susceptibility of A. actinomycetocomitans to antibiotics with maturation of the biofilm.

As sparse biofilm develop just after mechanical debridement and mature into denser biofilm if sufficient nutrients are available, it is important to emphasize that while antibiotic administration might be beneficial in assisting in the suppression of A. actinomycetocomitans in the periodontal pocket, effective mechanical removal of biofilm as part of the periodontal treatment is crucial prior to chemotherapy[17-20]. So the precise use of antibiotics after effective plaque control measures in LAP patients might enhance better treatment.

\section{Conclusion}

The concomminent use of antibiotics in the treatment of LAP should be made mandatory. Plaque sampling and antibiotic susceptibility tests should be a routine procedure in treating LAP patients to prevent overuse, misuse and development of resistant strains. Apart from the regular use of doxycycline, amoxicillin and metronidazole combination and other quinolone derivatives, studies on the beneficial effect of kanamycin and netilmycin in LAP patients should be done in future.

\section{References}

1. Armitage GC (1999) Development of a classification system for periodontal diseases and conditions. Ann Periodontol p. 4: 1.

2. Carranza's clinical period ontology, $\left(9^{\text {th }}\right.$ edn) WB Saunders Company, Philadelphia London, New York, St. Louis Sydney Toronto, USA.

3. Armitage GC, Cullinan MP (2010) Comparison of the clinical features of chronic and aggressive periodontitis. Periodontology 53:12-27.

4. Slots J, Reynolds HS, Genco RJ (1980) Actinobacillus actinomycetemcomitans human periodontal disease: a cross-sectional microbiological investigation. Infect Immun 29(3): 1013-1020.

5. Poveda-Roda R, Bagán JV, Sanchis-Bielsa JM, Carbonell-Pastor E (2007) Antibiotic use in dental practice. A review. Med Oral Patol Oral Cir Bucal 12(3): E186-E192.

6. Bokor-Bratić M, Brkanić T (2000) Clinical use of tetracyclines in the treatment of periodontal diseases. Med Pregl 53(5-6): 266-271

7. Rams TE, Feik D, Slots J (1992) Ciprofloxacin/metronidazole treatment of recurrent adult Periodontitis. Abstract. J Dent Res 71: 319.

8. Abinaya Prakasam, S Sugumari Elavarasu, Ravi Kumar Natarajan (2012) Antibiotics in the management of aggressive periodontitis. J Pharm Bioallied Sci 4(S2): S252-S25.

9. Van Winkelhoff AJ, Tijhof CJ, de Graaff J (1992) Microbiological and clinical results of metronidazole plus amoxicillin therapy in Actinobacillus actinomycetemcomitans-associated periodontitis. J Periodontol 63(1): 52-57.

10. Jorgensen MG, Slots J (2000) Practical antimicrobial periodontal therapy. Compend Contin Educ Dent 21(2): 111-114.

11. Weinstein L (1975) Antimicrobial agents: Penicillins and cephalosporins. In: Goodman LS, Gilman A, (Eds.). The Pharmacological Basis of Therapeutics. ( $5^{\text {th }}$ edn.). Macmillan, New York, USA.

12. Orit Oettinger-Barak, Stuart G Dashper, Deanne V Catmull, Geoffrey G Adams, Michael N (2013) Sela2, Eli E Machtei and Eric C Reynolds. Antibiotic susceptibility of Aggregatibacter actinomycetemcomitans JP2 in a biofilm. Journal of Oral Microbiology 5: 20320.

13. Sandhya Shrivastava, Sushruth Nayak, Prachi Nayak, Anika Vishwakarma Radhika NB, Bheemappa Bangennavar (2013) Antibiotic sensitivity against microbial profile of chronic and aggressive periodontitis subjects - a clinical trial. Chhattisgarh Journal of Health Sciences 1(1).

14. Walker C, Karpinia K (2002) Rationale for use of antibiotics in periodontics. J Periodontol 73(10): 1188-1196.

15. Veloo AC, Seme K, Raangs E, Singadji Z, Wekema-Mulder G, et al. (2012) Antibiotic susceptibility profiles of oral pathogen. Int J Antimicrob Agents 40(5): 450-454.

16. Takahashi N, Ishihara K, Kato T, Okuda K (2007) Susceptibility of Actinobacillus actinomycetemcomitans to six antibiotics decreases as biofilm matures. J Antimicrob Chemother 59(1): 59-65.

17. Bahar H, Torun MM, Demirci M, Kocazeybek B (2005) Antimicrobial resistance and b-lactamase production of clinical isolates of Prevotella and Porphyromonasspecies. Chemotherapy 51(1): 9-14.

18. Herrera D, Sanz M, Jepsen S, Needleman I, Roldán S (2002) A systematic review on the effect of systemic antimicrobials as an adjunct to scaling and root planing in periodontitis patients. J Clin Periodontol 29(S3): 136-159.

19. Rainer Buchmann, Rüdiger $F$ Müller, Achim Heinecke, Dieter $E$ Lange (2000) Actinobacillus actinomycetemcomitans in Destructive 
Periodontal Disease. Three-Year Follow-Up Results. Journal of Periodontology 71(3): 444-453.

\section{(C) (i) This work is licensed under Creative}

Submission Link: http://biomedres.us/submit-manuscript.php
20. Eva M Kulik, Lenkeit K, Chenaux S, Meyer J (2008) Antimicrobial susceptibility of periodontopathogenic bacteria. Journal of Antimicrobial Chemotherapy 61(5): 1087-1091.

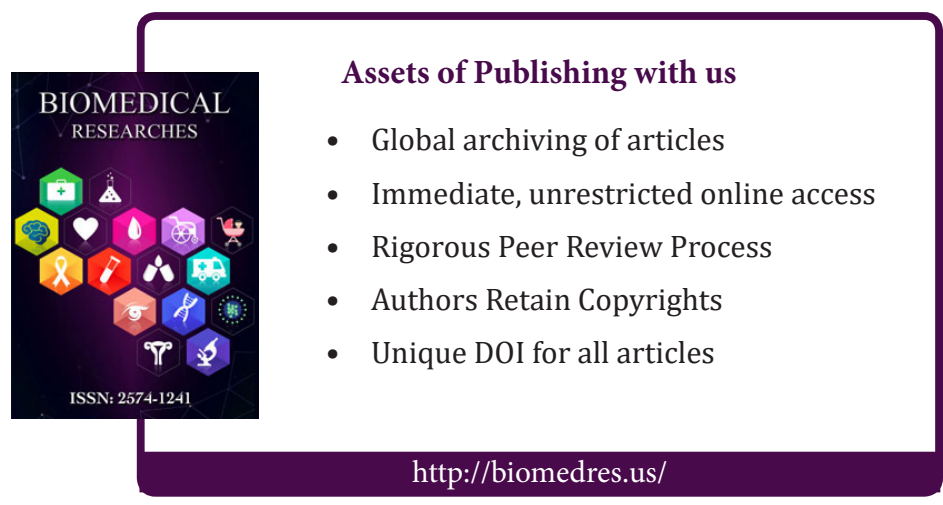

